
eISSN 2007-6835

\title{
Propiedades reológicas y mecánicas de un hormigón autocompactante con adición de nano-sílice y micro-sílice
}

\author{
E. Sánchez ${ }^{1}$, J. Bernal ${ }^{2}$, N. León ${ }^{3}$, A. Moragues ${ }^{3}$ \\ ${ }^{1}$ Dpto. de Ingeniería Agroforestal, Universidad Politécnica de Madrid; España. \\ ${ }^{2}$ Escuela de Ingeniería Mazatlán. Universidad Autónoma de Sinaloa. México. \\ ${ }^{3}$ Dpto. Ingeniería Civil: Construcción, Universidad Politécnica de Madrid; España.
}

\begin{abstract}
Información del artículo Artículo recibido el 22 septiembre de 2015, revisado bajo las políticas de publicación de la Revista ALCONPAT y aceptado el 15 de Diciembre de 2015. Cualquier discusión, incluyendo la réplica de los autores, se publicará en el tercer número del año 2016 siempre y cuando la información se reciba antes del cierre del segundo número del año 2016.
\end{abstract}

\section{(C) 2016 ALCONPAT Int.}

\section{Información Legal}

Revista ALCONPAT, Año 6, No. 1 ,

Enero - Abril 2016, es una publicación cuatrimestral de la Asociación

Latinoamericana de Control de Calidad,

Patología y Recuperación de la

Construcción, Internacional, A.C., Km.

6 , antigua carretera a Progreso, Mérida

Yucatán, C.P. 97310 ,

Tel.5219997385893,

alconpat.int@gmail.com, Página Web:

www.mda.cinvestav.mx/alconpat/revista

Editor responsable: Dr. Pedro Castro

Borges. Reserva de derechos al uso

exclusivo No.04-2013-011717330300-

203, eISSN 2007-6835, ambos

otorgados por el Instituto Nacional de

Derecho de Autor. Responsable de la

última actualización de este número,

Unidad de Informática ALCONPAT,

Ing. Elizabeth Sabido Maldonado, Km.

6, antigua carretera a Progreso, Mérida

Yucatán, C.P. 97310 , fecha de última
modificación: 30 de enero de 2016.

Las opiniones expresadas por los autores no necesariamente reflejan la postura del editor.

Queda totalmente prohibida la

reproducción total o parcial de los

contenidos e imágenes de la publicación

contenidos e imágenes de la
sin previa autorización de la

ALCONPAT Internacional A.C.

\section{RESUMEN}

El hormigón autocompactante es el resultado de diseñar mezclas de calidad con capacidad para asegurar su correcta colocación en estructuras fuertemente armadas en las cuales el proceso del vibrado resulta muy complicado y con riesgo de alterar la posición de las armaduras. Unido a las ventajas de este hormigón y debido a la mayor demanda de hormigones de altas prestaciones, se utiliza humo de sílice y, más recientemente, nanomateriales como adiciones. Principalmente nano-sílice. El objetivo de este trabajo es obtener hormigones autocompactantes con nano-sílice, humo de sílice y mezclas binarias de ambas adiciones que satisfagan la demanda de altas resistencias mecánicas y durables, determinando que la dosificación con mejores prestaciones es la que contiene $2.5 \%$ de nano y $2.5 \%$.de humo de sílice. Palabras clave: Autocompactante; nanosílice; humo de sílice; reología; propiedades mecánicas.

\section{ABSTRACT}

Self-compacting concrete is the result of designing a quality concrete with the capacity to ensure placement of reinforcement in heavily reinforced structures in which the process of vibrating is very complicated and risky by altering the position of the reinforcement. Together with the advantages of this concrete and due to the increased demand for high-performance concretes, the silica fume, and more recently, the nano-materials are used as additions, but mainly the nano-silica. The objective of this work is to obtain self compacting concrete with nano-silica, silica fume and binary mixtures of the two additions to meet the demand for high mechanical and durable resistance. The mix with better performance is that with $2.5 \%$ of nano-sílica and $2.5 \%$ silica fume.

Keywords: self-compacting concrete; nanosilica; silica fume; rheology; mechanical properties.

\section{RESUMO}

O Concreto Auto-adensável é o resultado da concepção de um concreto de qualidade com a capacidade para assegurar a colocação de reforço em estruturas fortemente armados em que o processo de vibração é muito complicado e arriscado por alterar a posição da armadura . Juntamente com as vantagens deste concreto e devido ao aumento da procura de concretos de alto desempenho, o fumo de sílica e mais recentemente, os nano-materiais são usados como adições . Principalmente a nano- sílica. O objetivo deste trabalho é a obtenção de concreto auto- adensável com nano- sílica , sílica ativa e misturas binárias das duas adições para atender a demanda de alta resistência mecânica e durável. A mistura com melhores desempenhos é aquela que contém 2,5\% de nano - sílica e 2,5\% de pó de sílica .

Palavras-chave: Auto-compactável; nano-sílica; sílica activa; reologia; propriedades mecánicas.

Autor de correspondencia: Elvira Sánchez (elvira.sanchez.espinosa@upm.es) 
Revista ALCONPAT, Volumen 6, Número 1, Enero - Abril 2016, Páginas 1 - 14

\section{INTRODUCCIÓN}

El hormigón autocompactante es el resultado de diseñar un hormigón de calidad con capacidad para asegurar su correcta colocación en estructuras fuertemente armadas en las cuales el proceso del vibrado resulta muy complicado y con riesgo de alterar la posición de las armaduras. El profesor Okamura y Ozawa (1996) inicia el desarrollo de dicho hormigón en la Universidad de Tokio a mediados de los 90, poniéndolo a punto en los años siguientes (Okamura, 1997, Okamura y Ouchi, 1999, Okamura, Ozawa, y Ouchi, 2000, Okamura, Maekawa, y Mishima, 2005). Este hormigón se caracteriza por una reducida relación agua/cemento, un alto contenido de finos, para lo cual se agrega filler calizo a la mezcla, un reducido contenido en árido grueso y la inclusión de aditivos superplastificante. Todo ello permite obtener un hormigón de alta fluidez, que por acción de su propio peso, consigue una buena consolidación sin exudación ni segregación (De la Peña, 2001, EHE-08, 2010). En la industria de los prefabricados las ventajas del uso de este hormigón son aún mayores, ya que aumenta la vida útil de los moldes debido a que la ausencia de vibrado permite que estos sean más ligeros y, por tanto, se reducen los gastos de mantenimiento.

Unido a las ventajas que presenta el hormigón autocompactante y como consecuencia del incremento en la demanda de hormigones de altas resistencias, se comienzan a utilizar nanomateriales como adiciones. Esto tiene como objeto conferir al material características especiales ya sea en su capacidad resistente o en su comportamiento durable. Por lo tanto, el hormigón que se pretende obtener se considera como un hormigón de altas prestaciones, ya que no solo debe satisfacer la demanda de altas resistencias mecánicas, sino que también debe cumplir con altas exigencias respecto a su durabilidad.

Son numerosas las nano-partículas investigadas, siendo las más usadas las de sílice, titanio, alúmina y hierro (Sanchez y Sobolev, 2010). La naturaleza del tipo de adición a elegir, depende de las propiedades que se quieran mejorar o conferir al material, en relación con la funcionalidad que se le quiera dar. La mayor concentración de estudios referentes a la incorporación de nanopartículas en el área de la ingeniería civil son las referentes a la nano- $\mathrm{SiO}_{2}$ (Kawashima, Hou, Corr, Shah, 2013). La mayoría de estudios coinciden en afirmar que la incorporación de dicha nano-partícula produce cambios microestructurales ya que es un activador de las reacciones puzolánicas (Bjornstrom, Martinelli, Matic, Borjesson, Panas, 2004). De forma similar al ya muy conocido humo de sílice o micro-sílice, la nano- $\mathrm{SiO}_{2}$ reacciona con el hidróxido de calcio $(\mathrm{Ca}$ $(\mathrm{OH})_{2}$ ), produciendo una mayor cantidad de gel CSH que densifica el material, con la consiguiente reducción de su permeabilidad (Said, Zeidan, Bassuoni, Tian, 2012) y control de la lixiviación del $\mathrm{Ca}^{2+}$ (Nazari y Riahi, 2010). El tamaño de la sílice adicionada produce cambios en el número y tamaño medio de los cristales de portlandita. Estos cambios microestructurales vienen asociados a cambios en las propiedades macroestructurales tanto de morteros como de hormigones tales como resistencia a compresión, módulo elástico (Yu, Spiesz, Brouwers, 2014; Zyganitidis, Stefanidou, Kalfagiannis, Logothetidis, 2011) y durabilidad León, Massana, Alonso, Moragues, SánchezEspinosa, 2014) entre otras.

Hay varios estudios sobre la influencia de nano y micro sílice en hormigones en las que se comparan distintos parámetros (Mondal, Shah, Marks, Gaitero, 2010; Borralleras, 2012; Craeye, Van Itterbeeck, Desnerck, Boel, De Schutter, 2014; Rong, Sun, Xiao, Jiang, 2015) pero hay pocos trabajos en donde se incluyan mezclas binarias de ambas adiciones en hormigones autocompactantes de altas prestaciones. Por tanto, en este trabajo se pretende estudiar la influencia de la nano-sílice, de la micro-sílice o humo de sílice así como adiciones de mezclas ternarias sobre las propiedades reológicas y mecánicas de hormigones autocompactantes con el fin de establecer las diferencias entre las distintas dosificaciones y poder determinar la mezcla con el mejor comportamiento frente a estas propiedades, tanto en estado fresco como en estado endurecido. 
Revista ALCONPAT, Volumen 6, Número 1, Enero - Abril 2016, Páginas 1 - 14

\section{PROCEDIMIENTO}

Para la fabricación de las mezclas se utilizó cemento portland CEM I 52,5 R (CP) según EN 1971 , cuyas propiedades están en la tabla 1 .

Tabla 1. Propiedades físico-químicas del cemento Portland (CP), nanoSi (nSi) y microSi (mSi).

\begin{tabular}{|c|c|c|c|c|c|c|c|c|c|c|c|}
\hline & $\mathrm{SiO}_{2}$ & $\mathrm{Al}_{2} \mathrm{O}_{3}$ & $\mathrm{Fe}_{2} \mathrm{O}_{3}$ & $\mathrm{CaO}$ & $\mathrm{MgO}$ & $\mathrm{SO}_{3}$ & $\mathrm{~K}_{2} \mathrm{O}$ & $\mathrm{Na}_{2} \mathrm{O}$ & $\begin{array}{c}\text { Pérd. al } \\
\text { fuego } \\
(\%)\end{array}$ & $\begin{array}{c}\text { Dens } \\
\left(\mathrm{g} / \mathrm{cm}^{3}\right)\end{array}$ & $\begin{array}{c}\text { Sup. } \\
\text { espec. } \\
\left(\mathrm{m}^{2} / \mathrm{g}\right)\end{array}$ \\
\hline $\mathrm{CP}$ & 19.20 & 6.07 & 1.70 & 63.41 & 2.56 & 3.38 & 0.2 & 0,33 & 2,09 & 3,5 & 0,42 \\
\hline $\mathrm{nSi}$ & 99.90 & - & - & - & - & - & - & - & 0,10 & 1,29 & 200 \\
\hline $\mathrm{mSi}$ & 94 & - & - & - & - & - & - & - & & & \\
\hline
\end{tabular}

La nanosílice (nSi) utilizada es Levasil 200/40\% en forma de dispersión acuosa con una riqueza del 40\%, una superficie específica de $200 \mathrm{~m}^{2} / \mathrm{g}$ y un tamaño de partícula de $15 \mathrm{~nm}$ (aprox) (véase Tabla 1). La microsílice o humo de sílice (mSi) utilizada es Elkem Microsilica MS 940 U, compuesta de esferas amorfas, submicrómicas, no porosas de $\mathrm{SiO}_{2}$ y pequeños aglomerados de éstos, con una superficie especifica de $15-30 \mathrm{~m}^{2} / \mathrm{g}$ y un tamaño medio de partícula de las esferas de aproximadamente 0,15 micras. Aunque algunas de las esferas pueden encontrarse individualmente, la mayoría de ellas forman aglomerados de partículas primarias con un rango típico de 0.1-1 micra (véase Tabla 1). Los aditivos utilizados fueron: superplastificante SIKA Viscocrete 5720 (SP) (polycarboxylato polímero) y aditivo modificador de viscosidad (MV) SIKA Stabilizer 4R. Los áridos empleados en las mezclas fueron: arena de rio menor a $4 \mathrm{~mm}$, grava rodada de 6 a $12 \mathrm{~mm}$ y filler calizo cuya granulometría cumple con la norma UNE 12620:2003+A1:2009, con un diámetro máximo de $63 \mu \mathrm{m}$.

Se diseñaron diez dosificaciones (véase Tabla 2). Todas las muestras mantuvieron una relación agua/material cementante de 0.36 . Las dosificaciones fueron: tres con $2.5 \%, 5 \%$ y $7.5 \%$ de nano$\mathrm{SiO}_{2}([\mathrm{nSi}]-2.5$; [nSi]-5; [nSi]-7.5), tres con $2.5 \%, 5 \%$ y $7.5 \%$ de micro-SiO $2([\mathrm{mSi}]-2.5 ;[\mathrm{mSi}]-5$; [mSi]-7.5), y tres con mezclas de ambas con los porcentajes de cada adición de 2.5\%/2.5\%, $5 \% / 2.5 \%$ y $2.5 \% / 5 \%$ de nano- $\mathrm{SiO}_{2}$ y micro- $\mathrm{SiO}_{2}$ respectivamente ([nmSi]-2.5/2.5; [nmSi] $-5 / 2.5$; [nmSi]-2.5/5) respecto al peso del cemento. Se diseñó una décima mezcla, sin ningún tipo de adición, que será considerada el hormigón de referencia.

Para evaluar las características de autocompactabilidad de los hormigones diseñados se realizaron, en estado fresco, los ensayos normalizados de acuerdo con la instrucción EHE-08 (2010). Dichos ensayos son el de escurrimiento o extensión de flujo (UNE-EN 12350-8), el ensayo del embudo en V (UNE-EN 12350-9), el ensayo de la caja en L (UNE-EN 12350-10) y el ensayo de escurrimiento con anillo japonés (UNE-EN 12350-12).

Comprobada la autocompactabilidad de la mezcla, se procedió a la fabricación de 12 probetas cilíndricas de $100 \mathrm{~mm}$ de diámetro y $200 \mathrm{~mm}$ de altura y 2 probetas normalizadas de $150 \mathrm{~mm}$ de diámetro y $300 \mathrm{~mm}$ de altura. Estas probetas se mantuvieron en el laboratorio durante $24 \mathrm{~h}$. Transcurrido ese tiempo, fueron desmoldadas y se sometieron a un curado durante 28 días dentro de una cámara a una temperatura de $20 \pm 2{ }^{\circ} \mathrm{C}$ y una humedad superior a $95 \%$, de acuerdo a la norma UNE-EN 12390-2. 
Revista ALCONPAT, Volumen 6, Número 1, Enero - Abril 2016, Páginas 1 - 14

Tabla 2. Dosificación de los hormigones estudiados

\begin{tabular}{|c|c|c|c|c|c|c|c|c|c|c|}
\hline $\begin{array}{l}\text { Componen } \\
\text { tes }\left(\mathrm{kg} / \mathrm{m}^{3}\right)\end{array}$ & U & 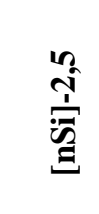 & $\frac{n}{\frac{1}{1}}$ & $\frac{n}{10}$ & 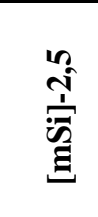 & $\frac{n}{\frac{1}{\bar{a}}}$ & 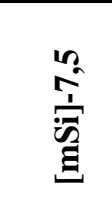 & 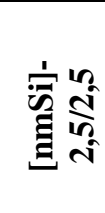 & 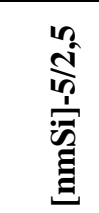 & $\begin{array}{l}\frac{n}{n} \\
\frac{n}{2} \\
\frac{1}{1} \\
\frac{1}{5} \\
\Xi \\
\Xi\end{array}$ \\
\hline Cemento & 450 & 450 & 450 & 450 & 450 & 450 & 450 & 450 & 450 & 450 \\
\hline \multirow{2}{*}{ Nanosílice } & \multirow{2}{*}{-} & 11,25 & 22,5 & 33,75 & \multirow[b]{2}{*}{ - } & \multirow{2}{*}{-} & \multirow{2}{*}{-} & 11,25 & 22,5 & 11,25 \\
\hline & & $2,5 \%$ & $5 \%$ & $7,50 \%$ & & & & $2,5 \%$ & $5 \%$ & $2,5 \%$ \\
\hline \multirow{2}{*}{ Microsílice } & \multirow{2}{*}{-} & \multirow{2}{*}{-} & \multirow[b]{2}{*}{ - } & \multirow[b]{2}{*}{ - } & 11,25 & 22,5 & 33,75 & 11,25 & 11,25 & 22,5 \\
\hline & & & & & $2,5 \%$ & $5 \%$ & $7,50 \%$ & $2,5 \%$ & $2,5 \%$ & $5 \%$ \\
\hline Agua & 162 & 166,0 & 170,1 & 174,1 & 166,0 & 170,1 & 174,1 & 170,1 & 174,1 & 174,1 \\
\hline $\begin{array}{l}\text { Filler } \\
\text { calizo }\end{array}$ & 100 & 100 & 100 & 100 & 100 & 100 & 100 & 100 & 100 & 100 \\
\hline Arena & 1160 & 1160 & 1160 & 1160 & 1160 & 1160 & 1160 & 1160 & 1160 & 1160 \\
\hline Grava 6/12 & 585 & 585 & 585 & 585 & 585 & 585 & 585 & 585 & 585 & 585 \\
\hline SP $(\%)^{*}$ & 2 & 3,30 & 4 & 6 & 2,30 & 2,50 & 2,70 & 3,60 & 4,80 & 3,90 \\
\hline MV $(\%)^{*}$ & 0,15 & 0,15 & 0,15 & 0,15 & 0,15 & 0,15 & 0,15 & 0,15 & 0,15 & 0,15 \\
\hline
\end{tabular}

(*) Porcentaje en peso de cemento.

Para caracterizar los hormigones de acuerdo a sus propiedades mecánicas se ensayaron 3 probetas a resistencia a compresión a los 7, 28 y 90 días de su fabricación, según la norma UNE-EN 123903. El tamaño máximo del árido, inferior a $12 \mathrm{~mm}$, es lo que permitió su uso para dicho ensayo (Fernandez, 2013).También, y a 28 días de curado, se procedió a determinar la resistencia a tracción indirecta mediante el ensayo de 2 probetas de 100x200 mm de acuerdo a la norma UNEEN 12390-6:2001. Las 2 probetas de 150x300 mm se ensayaron a esa misma edad para determinar el módulo de elasticidad de acuerdo a la norma UNE-EN 83316:1996. Dichos ensayos se realizaron en una prensa IBERTEST, con una capacidad máxima de $1.500 \mathrm{kN}$. Para determinar el módulo de elasticidad se midieron las deformaciones en las probetas normalizadas mediante un transformador diferencial de variación lineal de la casa IBERTEST con un sistema de adquisición de datos controlado por ordenador. Las cargas se incrementaron uniformemente a la velocidad de $0.2 \mathrm{MPa} / \mathrm{s}$, durante 3 ciclos sucesivos de carga y descarga hasta un $40 \%$ de la resistencia de compresión. Los valores de resistencia a compresión, tracción indirecta y módulo de elasticidad son el valor medio de los resultados obtenidos en su correspondiente ensayo.

La probeta restante se utiliza para la caracterización microestructural de las mezclas. Para ello se realizó, para todas las edades y mezclas diseñadas, un análisis termogravimétrico (ATG) a 7, 28 y 90 días, según la norma ASTM E1131:(2008).

Para este ensayo se empleó una muestra en polvo, con un tamaño de partícula inferior a 0,5 mm. Para conseguir estas características se utilizó una muestra de $20 \mathrm{~mm}$ obtenida de la probeta después de haber eliminado los $20 \mathrm{~mm}$ más externos. La muestra obtenida se pasó por una moledora de mandíbulas para obtener partículas de tamaños inferiores a los $8 \mathrm{~mm}$. Estas partículas se colocaron en un desecador conectado a una bomba de vacío, que garantizó una presión de vacío entre 1 y $5 \mathrm{kPa}$. Se mantuvo en el desecador durante $45 \mathrm{~min}$. Posteriormente, se sumergió, durante 24 horas, en isopropanol al 95\%, con objeto de detener los procesos de hidratación en el hormigón. Posteriormente, se secó en estufa a $40^{\circ} \mathrm{C}$ durante un tiempo mínimo de 72 horas, para garantizar el completo secado de la muestra.

Previo a la realización del ensayo, las partículas se molieron en un mortero manual de acero, terminándose de refinar el grano en un molino de ágata, Retsch RM 200. El proceso finaliza 


\section{Revista ALCONPAT, Volumen 6, Número 1, Enero - Abril 2016, Páginas 1 - 14}

mediante el cribado del polvo a través de un tamiz de $0.5 \mathrm{~mm}$. La masa molida, de aproximadamente un peso de $300 \mathrm{~g}$, se agitó, en bolsa de cierre hermético, para homogeneizarla. Se hizo un cuarteo de la muestra, dividiendo el total en 8 partes y tomando una porción de cada parte hasta completar dos muestras de $2 \mathrm{~g}$, que se colocaron en estufa a $40^{\circ} \mathrm{C}$ durante 7 días para estabilizar la masa. El equipo utilizado para el análisis termogravimétrico fue un analizador térmico simultáneo, marca SETARAM, modelo LABSYS EVO, con una balanza de precisión de $0.1 \mu \mathrm{g}$. Para el ensayo se empleó una muestra de aproximadamente $55 \mathrm{mg}$, que se sometió a una rampa de calentamiento dinámico que varío entre $40^{\circ} \mathrm{C}$ y $1.100^{\circ} \mathrm{C}$ con una velocidad de calentamiento de $10^{\circ} \mathrm{C} / \mathrm{min}$. Se utilizaron crisoles de alúmina, como material de referencia $\alpha$ alúmina $\left(\alpha-\mathrm{Al}_{2} \mathrm{O}_{3}\right)$, previamente calcinada a $1.200^{\circ} \mathrm{C}$, y una atmósfera de ensayo de nitrógeno $\left(\mathrm{N}_{2}\right)$. Este análisis permitió determinar la cantidad de gel CSH y de portlandita $\left(\mathrm{Ca}(\mathrm{OH})_{2}\right.$ presente en cada hormigón. Para ello se determinaron los valores de las pérdidas de agua de gel que se obtuvieron sometiendo a la muestra a temperaturas entre $105^{\circ} \mathrm{C}$ y $400^{\circ} \mathrm{C}$ aproximadamente. Las pérdidas de agua de portlandita libre se obtuvieron en un rango de temperatura entre $400^{\circ} \mathrm{C} \mathrm{y}$ $600^{\circ} \mathrm{C}$ aproximadamente.

\section{RESULTADOS Y DISCUSIÓN}

\subsection{Propiedades reológicas}

En la 0 se observa la inspección visual de las 10 mezclas, donde en ningún caso se presentó segregación ni exudación de la lechada. Este resultado es contrario al obtenido por Dubey y Kumar (2012) que afirman que con cantidades superiores al 2\% de un SP de tipo carboxilato, similar al utilizado en este estudio, se observa segregación de la mezcla.

Sin embargo, la evaluación visual de las mezclas mostró una gran cantidad de burbujeo en aquellas que contenían nSi. Este fenómeno es atribuido a la cantidad de SP que se utiliza para obtener la docilidad necesaria, tal como plantea Borrelleras Mas (2012).

En el caso de este estudio no se realizó un ensayo de aire ocluido a las mezclas. Sin embargo, Yu et al. (2014), realizaron ensayos en hormigones de altas prestaciones con contenidos de nanosílice entre un $1 \%$ hasta el $5 \%$ de adición con la cantidad de SP constante, y obtienen un aumento exponencial del contenido de aire ocluido, cuanto mayor es la cantidad de nSi, lo que permite concluir que tanto la cantidad de SP como la nSi, traen como consecuencia un aumento en la cantidad de aire ocluido.

Por otra parte, para evaluar si esta cantidad de aire podría eliminarse de las mezclas, se repitieron las amasadas con adición de nSi y se realizó un vibrado en mesa vibradora donde se observó que las burbujas de aire no salían a la superficie, y que la mezcla se movía de forma homogénea dentro de los moldes, formado una única masa que se movía uniformemente. Además, la capa superficial tenía una apariencia plastica que no permitía la expulsión de dichas burbujas. Posteriormente se realizaron ensayos de resistencia a compresión y PIM a estas mezclas vibradas, y no se observaron, en ningún caso, diferencias significativas, por lo que los valores de las mismas no se muestran en este estudio.

La propuesta de los autores de este estudio para mitigar la cantidad de burbujeo es dar mayor energía de mezclado a los hormigones, y aumentar el tiempo de mezclado en la amasadora, logrando así que esta cantidad de burbujas escapen por los movimientos mecánicos del amasado y obtener una mayor docilidad en las mezclas. También se recomienda la utilización, junto con el policarboxilato, de un polietileno condensado que actúe como desespumante. 
Revista ALCONPAT, Volumen 6, Número 1, Enero - Abril 2016, Páginas 1 - 14

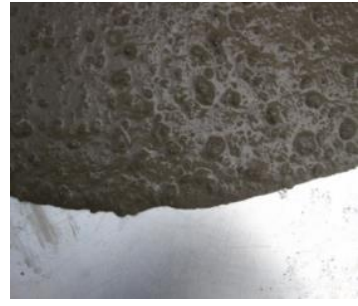

$\mathrm{HAC}[\mathrm{nSi}]-2,5$

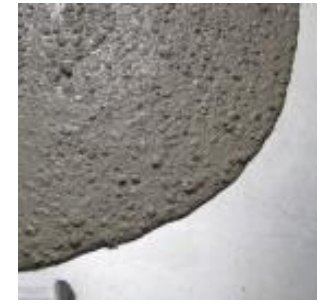

HAC

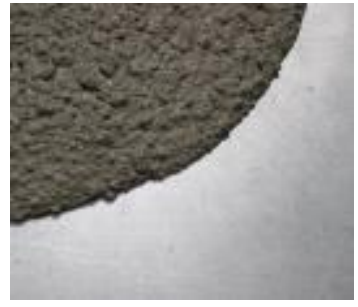

$\mathrm{HAC}[\mathrm{nSi}]-5$
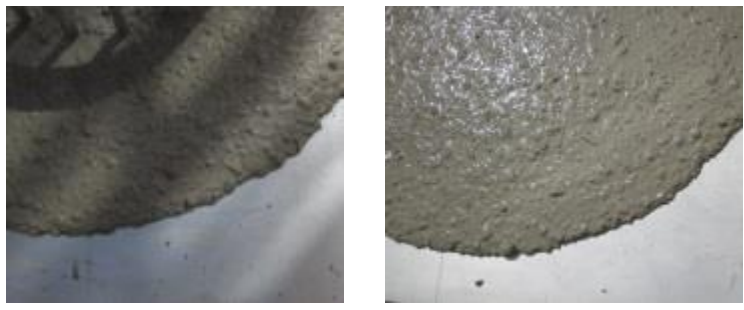

$\mathrm{HAC}[\mathrm{mSi}]-2,5 \quad \mathrm{HAC}[\mathrm{nmSi}]-2,5 / 2,5$

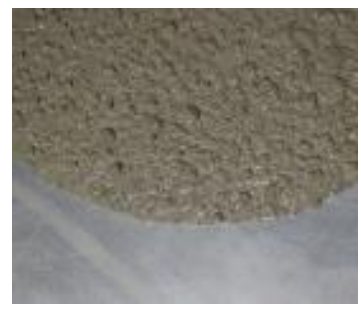

$\mathrm{HAC}[\mathrm{nSi}]-7,5$

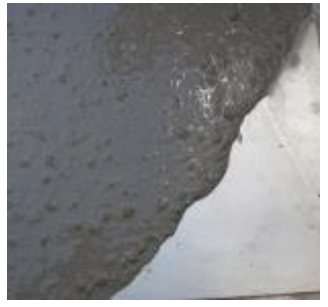

$\mathrm{HAC}[\mathrm{mSi}]-5 \quad \mathrm{HAC}[\mathrm{nmSi}]-5 / 2,5$

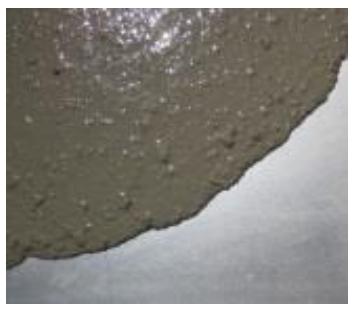

$\mathrm{HAC}[\mathrm{mSi}]-7,5 \quad \mathrm{HAC}[\mathrm{nmSi}]-2,5 / 5$
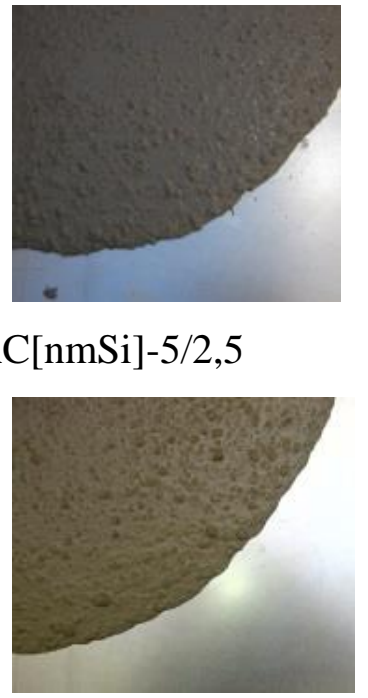

Figura 1. Aspecto del borde de las diferentes mezclas diseñadas, en estado fresco, después del ensayo de escurrimiento

Los valores obtenidos en los distintos ensayos para verificar la autocompactbilidad de las mezclas, de acuerdo a la EHE-08 (2010), se recogen en las Figuras 2, 3, 4 y 5. Estas presentan el diámetro de escurrimiento de las mezclas $\left(\mathrm{d}_{\mathrm{f}}\right)$ y el SP usado para su fabricación (Figura 2), el tiempo $\mathrm{T}_{\mathrm{V}}$ de paso por el embudo en V (Figura 3), la capacidad de paso por la caja en L $\left(\mathrm{C}_{\mathrm{bl}}\right)$ (Figura 4 ) y el diámetro de escurrimiento con anillo japonés $\left(\mathrm{d}_{\mathrm{jf}}\right)$ (Figura 5$)$.

En la figura 2 y de forma general, se observa que el diámetro de escurrimiento se encuentra entre $550 \mathrm{~mm}$ y $850 \mathrm{~mm}$, por lo que todas las mezclas cumplen con dicho parámetro. Además, no presentan segregación ni exudación, a pesar de tener elevadas cantidades de SP e incluso, para la mezcla [nSi]-7.5, superiores a lo permitido por la Instrucción Española del Hormigón Estructural (EHE-08) que es de un 5\%. Estos resultados contradicen a Dubey y Kumar (2012) que afirman que con cantidades superiores al $2 \%$ de un SP de tipo carboxilato, similar al utilizado en este estudio, se observa segregación de la mezcla. Incluso hay autores (Borralleras, 2012) que defienden que la cantidad máxima de SP queda determinada cuando el aditivo deja de producir mejoras reológicas en el material en estado fresco. 
Revista ALCONPAT, Volumen 6, Número 1, Enero - Abril 2016, Páginas 1 - 14

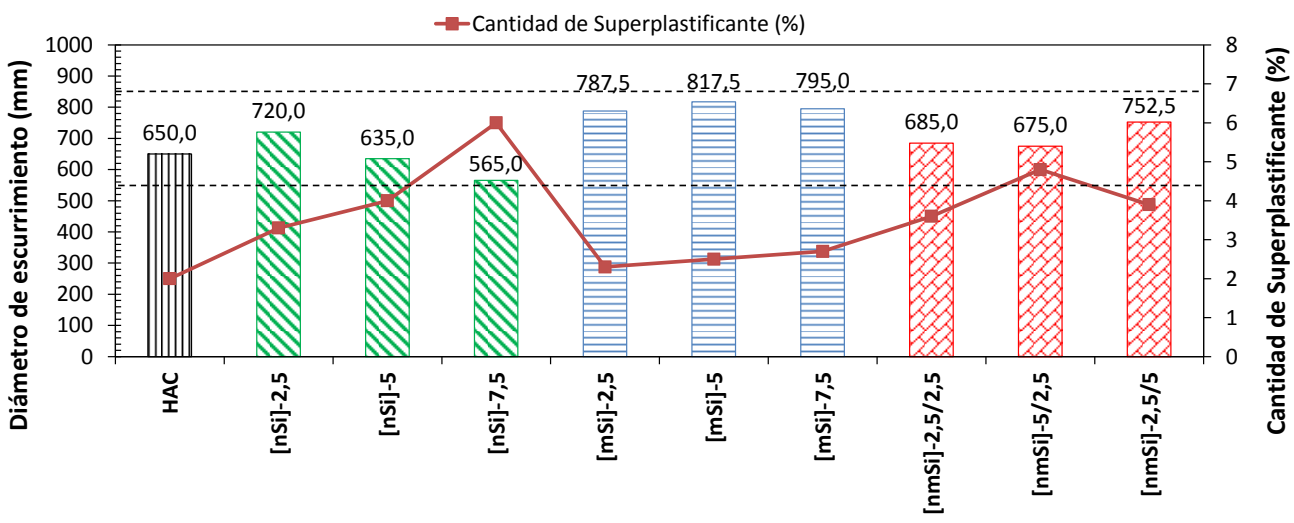

Figura 2. Diámetro de escurrimiento $\left(\mathrm{d}_{\mathrm{f}}\right)(\mathrm{mm})$ y cantidad de superplastificante.

También se puede observar la existencia de una clara influencia del tipo de adición. Así, las mezclas con adición de nSi muestran una disminución del diámetro de escurrimiento a medida que aumenta dicha adición. Esto se traduce en una menor docilidad de la mezcla, aun cuando hay un incremento hasta el 6\% en la cantidad de SP, superior al recomendado por la EHE. Este fenómeno no se produce cuando se utiliza $\mathrm{mSi}$ como adición. En este caso, existe muy poca variabilidad en el diámetro, que se encuentra alrededor de $800 \mathrm{~mm}$. Por último, en las mezclas binarias, se observa como la nSi compromete la docilidad del material, siendo necesario aumentar la cantidad de SP al aumentar la cantidad de nSi que se incorpore. Jalal et al. (2012) afirman que tanto la nSi como la mSi mejoran la consistencia del hormigón autocompactante, pero no entran a valorar el aumento de dicha consistencia con respecto al contenido ni tipo de estas. Sin embargo, constatan que cantidades de nSi del $2 \%$ respecto al peso de cemento no varían sustancialmente el diámetro de escurrimiento y si lo hacen las mezclas con un $10 \%$ de $\mathrm{mSi}$ y $2 \%$ de nSi. Por otra parte esta cantidad de aditivo produce un importante burbujeo que es directamente proporcional a la cantidad agregada (Nazari y Riahi, 2010). Estas burbujas pueden dar lugar a la formación de poros no interconectados en el material endurecido, que pueden comprometer las características resistentes de este hormigón por lo que se recomienda la utilización, junto con el policarboxilato, de un polietileno condensado que actúa como desespumante (Jalal et al., 2012).

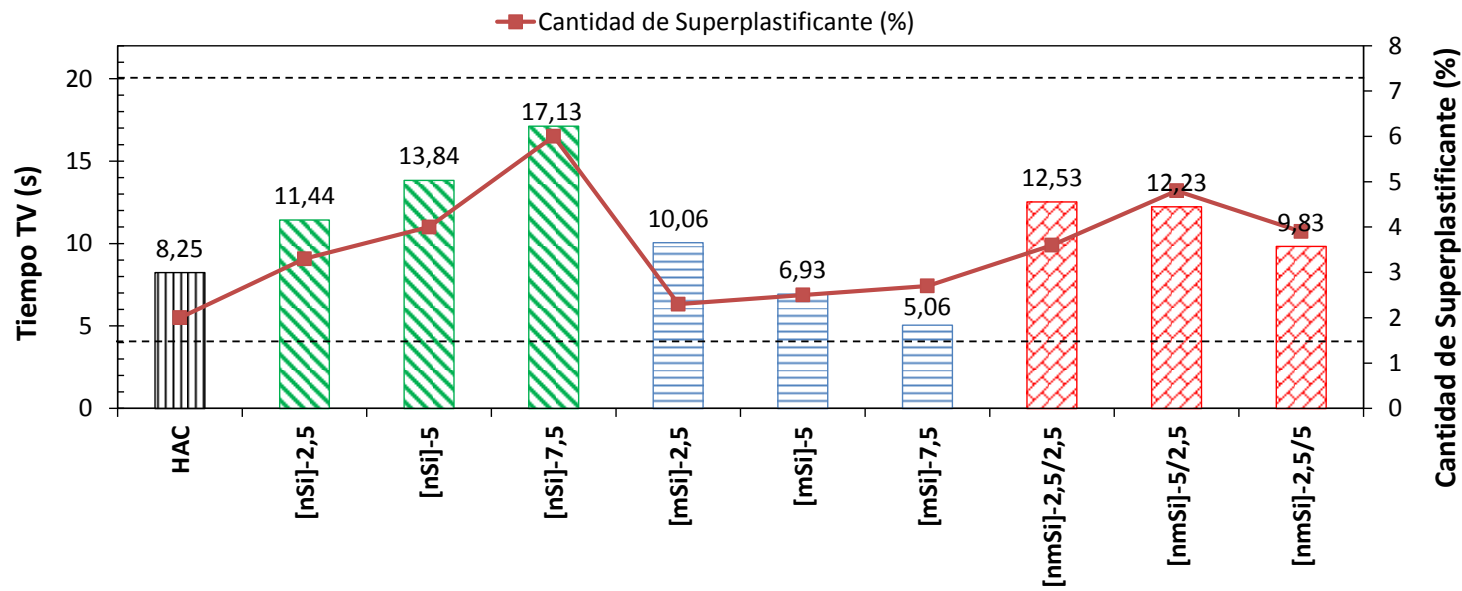

Figura 3. Tiempo (TV) de paso (s) por el embudo en V y cantidad de superplastificante.

En la figura 3 se muestra el tiempo TV de paso por el embudo en V. También para este parámetro se cumplen los requerimientos de la EHE-08 en cuanto a autocompactabilidad, ya que los valores se encuentran entre 4 s y 20 s. 
Revista ALCONPAT, Volumen 6, Número 1, Enero - Abril 2016, Páginas 1 - 14

En ella, se observa que el comportamiento de las mezclas es claramente diferente. Las que contienen solo nSi incrementan TV a medida que se incrementa el contenido en nSi, es decir, se densifican, mientras que en las mezclas con $\mathrm{mSi}$, el efecto es contrario, reduciéndose TV hasta 5.06 segundos cuando el contenido de $\mathrm{mSi}$ es del $7.5 \%$ respecto a la cantidad de cemento. En ambos casos es necesario un incremento de SP, siendo muy superior la cantidad de éste en las dosificaciones con $\mathrm{nSi}$. Por lo tanto, se puede afirmar que en las mezclas con $\mathrm{nSi}$, con una cantidad similar de $\mathrm{SP}$, se logran $\mathrm{d}_{\mathrm{f}}$ similares aunque la mezcla se hace cada vez más liquida, por la acción de la mayor cantidad de SP en ellas. Las mezclas binarias presentan un comportamiento poco concluyente, ya que a mayor contenido en $\mathrm{nSi}$ mayor exigencia en SP aunque su TV es similar, siendo más viscosa la mezcla al aumentar la cantidad de mSi aunque la demanda de SP es menor. Estos resultados discrepan de los obtenidos por Jalal et al. (2012) quienes obtienen un tiempo de paso, por el embudo en $\mathrm{V}$, mínimo de $4 \mathrm{~s}$ para la mezcla con $500 \mathrm{~kg} / \mathrm{m}^{3}$ y un $2 \%$ de $\mathrm{nSi}$ y máximo $(12 \mathrm{~s})$ para la mezcla binaria $10 \% \mathrm{mSi}+2 \% \mathrm{nSi}$ y $400 \mathrm{~kg} / \mathrm{m}^{3}$. Las diferencias en los contenidos de cemento podrían ser las responsables de estas notables diferencias en los resultados ya que un incremento en la cantidad de cemento mejora todas las propiedades reológicas debido a un incremento en el volumen de la pasta (Jalal et al., 2012).

En la figura 4 se presentan la medida de la capacidad de paso $\mathrm{C}_{\mathrm{bl}}$ encontrándose todos los hormigones con valores comprendidos entre los valores límite de 0.75 y 1 . A pesar de ello, se vuelve a observar un comportamiento diferente según el tipo de adición. Así, la capacidad de paso para mezclas con nSi experimenta un decrecimiento al incrementarse la cantidad de nSi.

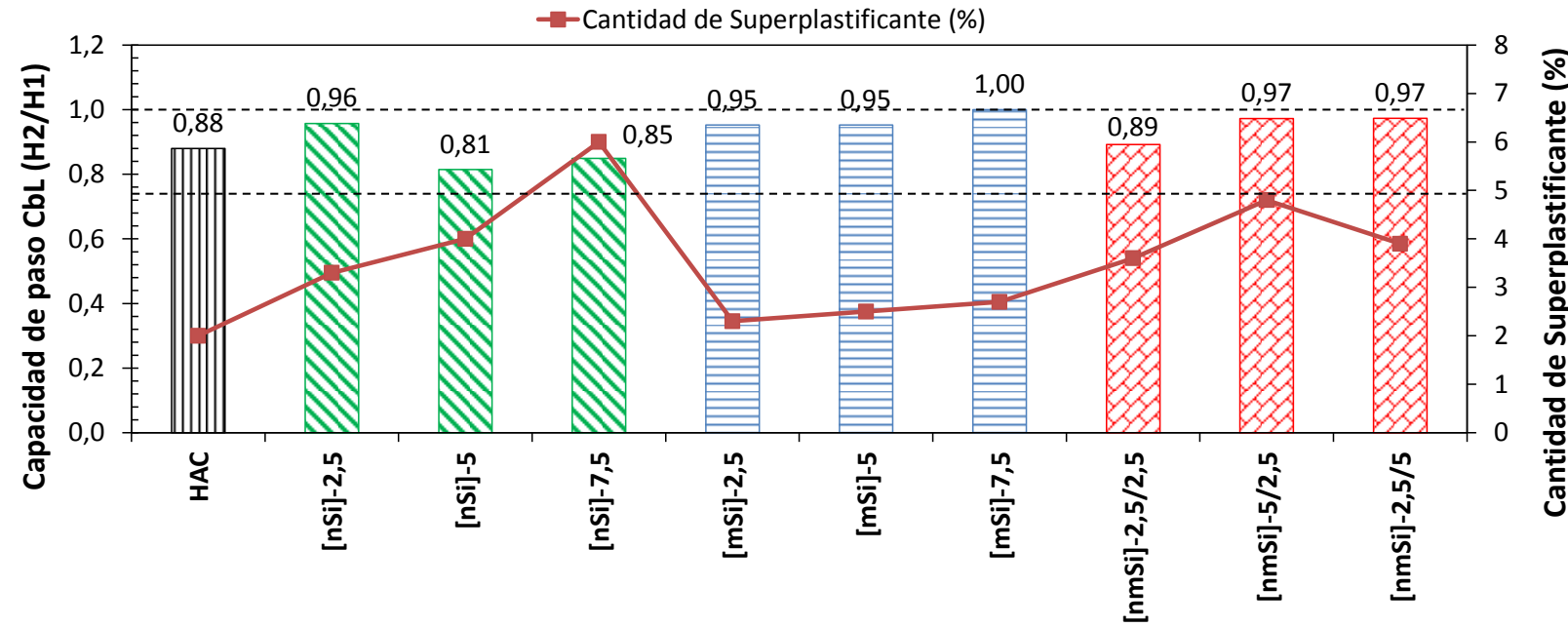

Figura 4. Capacidad de paso por la caja en L y cantidad de superplastificante.

Además, este parámetro proporciona información sobre la capacidad de autonivelación de los hormigones (EHE-08, 2010), siendo menos autonivelantes cuanto mayor es el contenido de nSi. Sin embargo, los valores permanecen prácticamente constantes tanto para las dosificaciones con $\mathrm{mSi}$, como para las mezclas con adiciones binarias. Cabe destacar, la muy buena capacidad autonivelante del hormigón que contiene un total de $7.5 \%$ de $\mathrm{mSi}$ con un valor de la unidad. Este buen comportamiento pueda deberse a que esta mezcla es la que presenta la mínima velocidad de paso PV, es decir, es la mezcla más líquida de todas las estudiadas.

En la figura 5, se muestra el valor del diámetro de escurrimiento con anillo japonés $\left(\mathrm{d}_{\mathrm{jf}}\right)$. La EHE08 limita el valor de este parámetro en función del diámetro de escurrimiento $\left(\mathrm{d}_{\mathrm{f}}\right)$ obtenido en la misma mezcla, teniéndose que cumplir que su diferencia sea inferior a $50 \mathrm{~mm}$, que se cumple para la totalidad de las mezclas diseñadas. 
Revista ALCONPAT, Volumen 6, Número 1, Enero - Abril 2016, Páginas 1 - 14

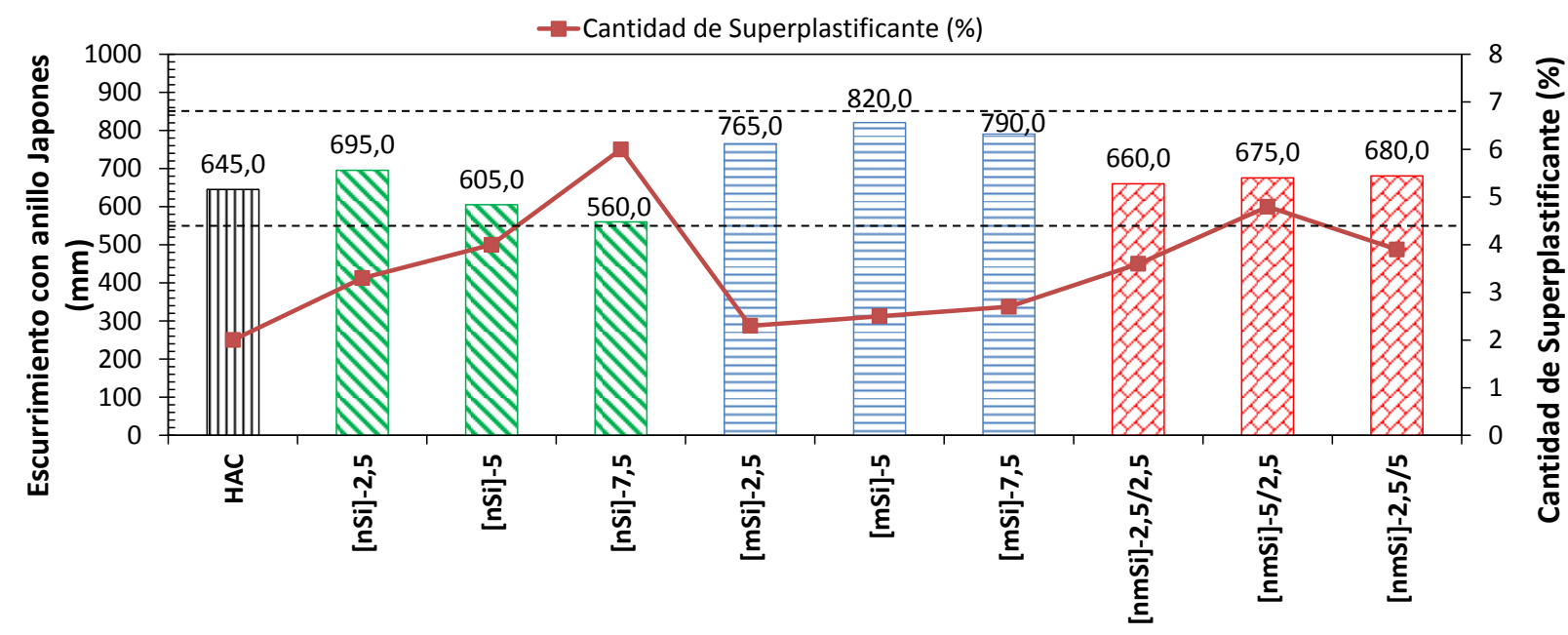

Figura 5. Diámetro de escurrimiento con anillo japonés (cm) y cantidad de superplastificante.

Al observar la figura 5 destaca que los hormigones con adición de nSi tienen un comportamiento similar a la del ensayo de escurrimiento $\left(\mathrm{d}_{\mathrm{f}}\right)$, aunque con valores inferiores, puesto que con él se determina la dificultad que puede tener el hormigón al paso con obstáculos. Así, a medida que es mayor la cantidad de nSi menor es el valor de este parámetro. En hormigones con mSi se dan los valores más altos. En las mezclas con adiciones binarias los valores no presentan diferencias significativas entre sí, ni con el obtenido en [nSi]-2.5. Esto indica que es el contenido de nSi quien gobierna estas mezclas, teniendo que aumentar el contenido de SP, cuando aumenta en contenido en nSi. Una observación importante, en la fabricación de las mezclas, fue que los hormigones con adición de nSi obtuvieron un notable aumento en la velocidad de fraguado, donde sin importar la cantidad de SP añadida, el comienzo del fraguado se producía a los pocos minutos de la fabricación. Esto dificultaba la manipulación de la mezcla y por tanto, su puesta en obra. Sin embargo, este fenómeno, puede ser una ventaja en la industria de los prefabricados, en las que un fraguado rápido puede ser beneficioso, debido a la posibilidad de desmoldar en tiempos más cortos. Este hecho coincide con lo estudiado por Bjornstrom et al. (2004) que afirma que la nSi es un activador de las reacciones puzolánicas.

\subsection{Propiedades mecánicas}

\subsubsection{Resistencia a compresión}

La resistencia a compresión de las distintas dosificaciones se presenta en la Figura 6. En esta, se observa que los hormigones con nSi tienen resistencias mayores a las obtenidas en el hormigón de referencia. Además, dicha resistencia aumenta a medida que aumenta la cantidad de adición.

En el caso de los hormigones con $\mathrm{mSi}$, las resistencias a compresión son ligeramente mayores al hormigón de referencia, con incrementos notables a los 7 días y moderados para el resto de edades. Por último, y en el caso de las mezclas con adiciones binarias, no hay un comportamiento consistente que sugiera la preponderancia de uno de los tipos de adición. Sin embargo, las resistencias más altas se consiguen con la mezcla [nmSi]-2.5/2.5, por lo que los autores consideran que la resistencia a compresión no solo depende del tamaño de partícula y de la cantidad de adición sino también de la distribución granulométrica de todos los componentes de la mezcla. Por lo que cuanto mayor continuidad en la granulometría de los componentes de la mezcla mayor será su compactación y por lo tanto, mayor su capacidad resistente.

En estas mezclas, los valores de resistencia a compresión son de $82.17 \mathrm{MPa}$ a los 28 días de curado y de $86.87 \mathrm{MPa}$ a los 90 días. Esto supone un incremento de un $36 \%$ con respecto a los 


\section{Revista ALCONPAT, Volumen 6, Número 1, Enero - Abril 2016, Páginas 1 - 14}

valores obtenidos por el hormigón de referencia a los tiempos de curado de 28 y 90 días respectivamente.

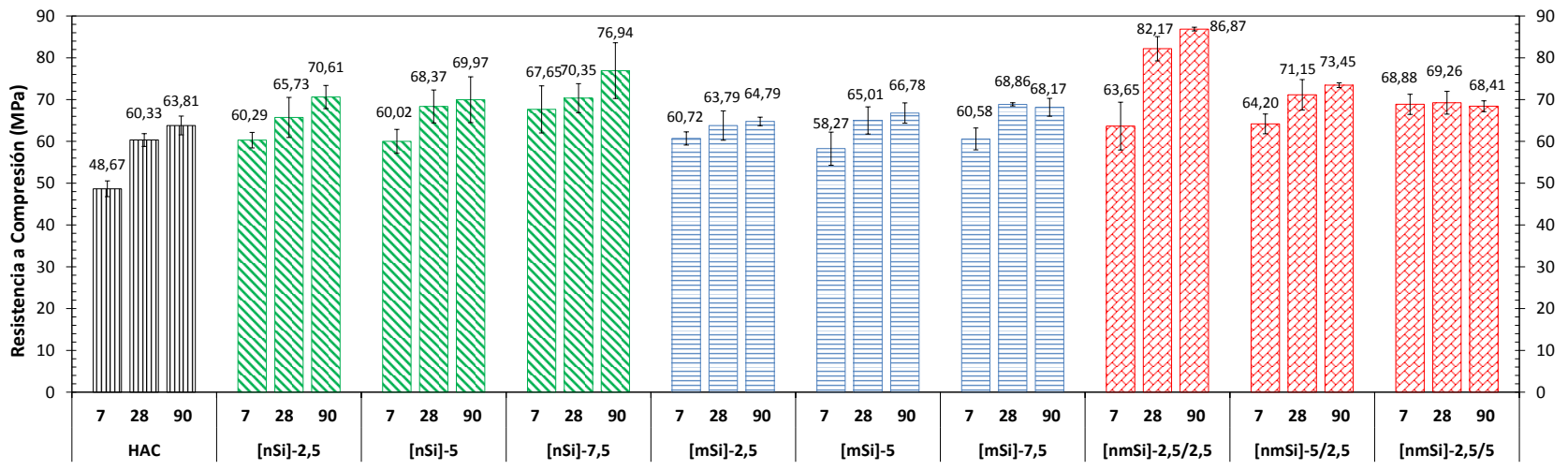

Figura 6. Resistencia a Compresión (MPa)

\subsubsection{Resistencia a tracción indirecta y módulo de elasticidad}

En las figura 7 y 8 se presentan los resultados de resistencia a tracción indirecta y del módulo de elasticidad.

El valor de la tracción indirecta en hormigones con adiciones es significativamente mayor al valor del hormigón de referencia, a pesar de que estas sean diferencias pequeñas. Sin embargo no existen diferencias significativas entre las mezclas con adiciones. Es decir, el tamaño y contenido de las distintas adiciones contenidas en las diferentes mezclas no cambian significativamente esta propiedad.

En cuanto al módulo de elasticidad se observan valores significativamente menores para las mezclas que contienen $\mathrm{nSi}$. En el resto de los hormigones, los valores obtenidos son similares no siendo significativos entre ellos ni respecto al hormigón de referencia.

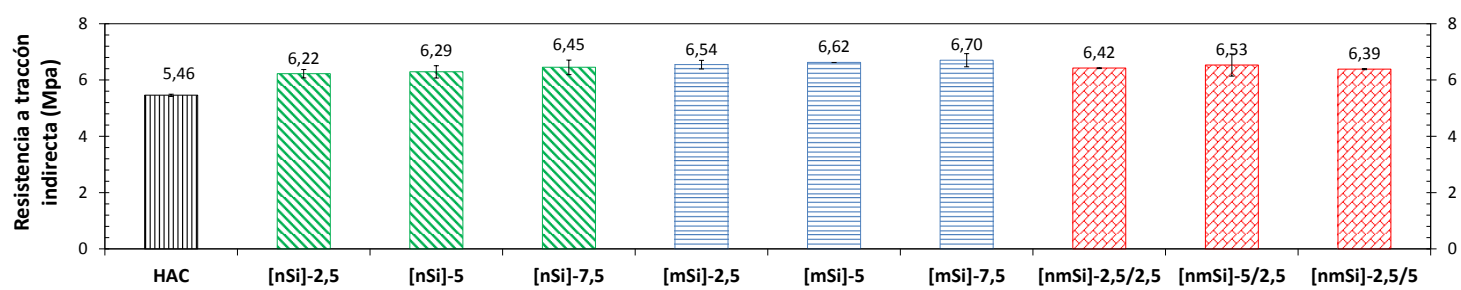

Figura 7. Resistencia a tracción indirecta $(\mathrm{MPa})$

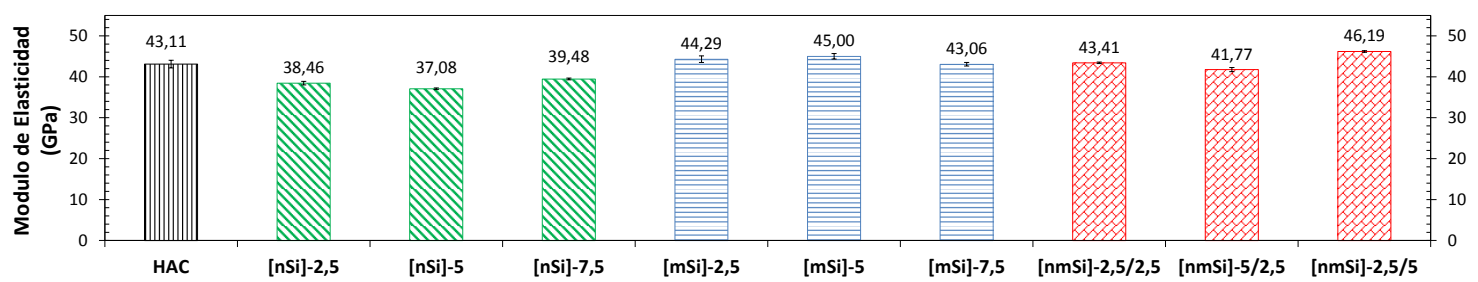

Figura 8. Módulo de elasticidad (GPa)

\subsection{Caracterización microestructural. Análisis termogravimétrico}

Los resultados del análisis termogravimétrico de las diez dosificaciones se muestran en las Figuras 9, 10 y 11 en la que se presentan la relación entre las pérdidas de agua de gel y de agua de portlandita libre a los 7, 28 y 90 días de curado, respectivamente. 


\section{Revista ALCONPAT, Volumen 6, Número 1, Enero - Abril 2016, Páginas 1 - 14}

En general, los valores obtenidos permiten afirmar que, a todas las edades, los mayores valores de dicha relación son los obtenidos en los hormigones con nSi. Esto pone de manifiesto que la formación de gel secundario o tobermorita es mayor lo que conlleva la menor presencia de portlandita.

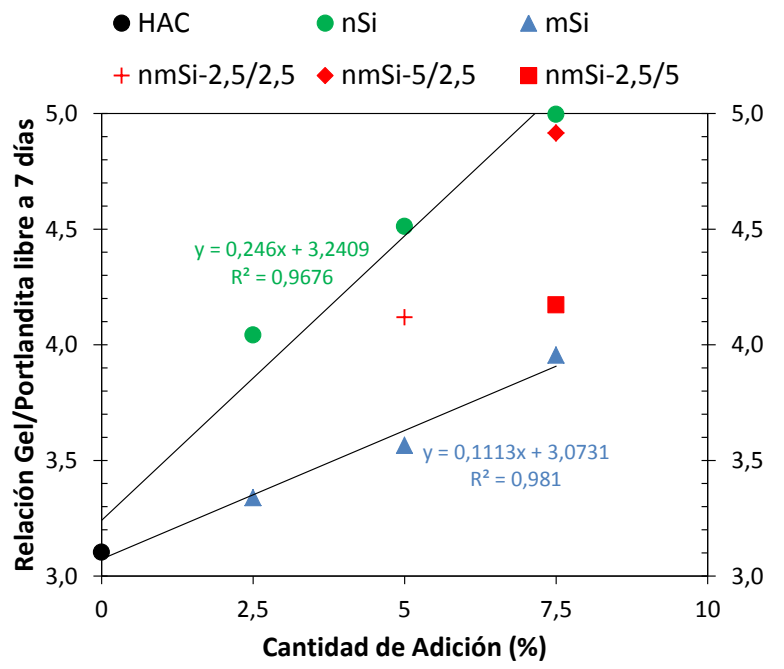

Figura 9. Relación gel/portlandita libre a 7 días de curado

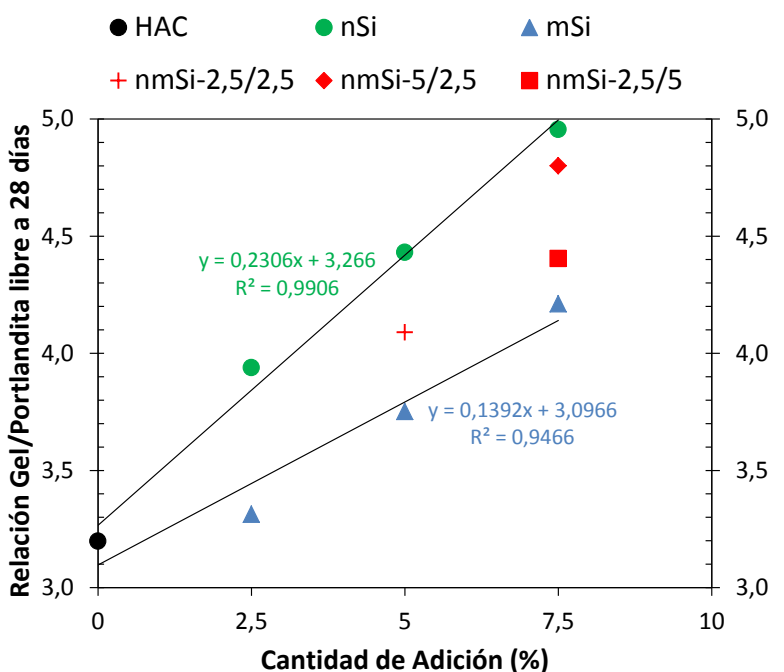

Figura 10. Relación gel/portlandita libre a 28 días de curado 


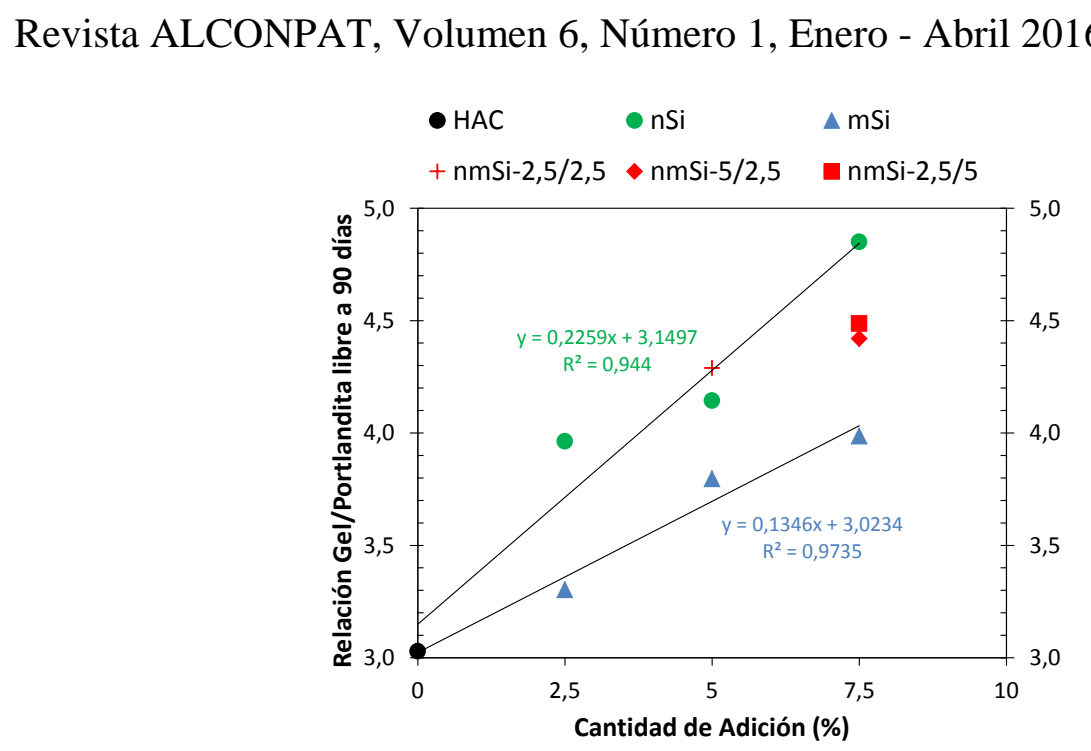

Figura 11. Relación gel/portlandita libre a 90 días de curado

Así mismo, en el caso de los hormigones con $\mathrm{mSi}$, las relaciones presentan valores menores coincidiendo con lo obtenido por Mondal et. al. (2010), Zyganitidis et al. (2011), Jalal et al. (2012) y León et al. (2014). En ambos casos, la relación aumenta cuando lo hace el contenido de la adición. Como anteriormente se indicó, los hormigones con combinación de adiciones no presentan un comportamiento concluyente. El comportamiento parece definido por el mayor contenido de cada una de las adiciones.

Por otra parte, es importante destacar que a medida que transcurre el tiempo de curado, las mezclas con nSi muestran un descenso en los valores de la relación gel/portlandita libre, fenómeno que se evidencia por la reducción en las pendientes de las rectas. Dicho fenómeno se puede observar, de forma progresiva en las Figura 9, 10 y 11. Esto sugiere que la formación de geles se ralentiza con la edad de curado, siendo en edades tempranas cuando mayor actividad puzolánica se observa (Jalal et al., 2012). Esto es concordante con los valores de resistencia a compresión a los 7 días de curado. Sin embargo, en los hormigones con $\mathrm{mSi}$, los valores de la relación gel/portlandita libre aumentan a medida que aumenta el tiempo de curado. Un menor tamaño de particula acelera la activación de la reacción puzolánica

$\mathrm{Al}$ analizar los hormigones que contienen mezclas binarias destaca que en las primeras edades las mezclas mixtas se comportan de manera similar a como lo hacen las mezclan que contienen un porcentaje de adición similar a la suma de los dos, pero en cada caso próximas a la adición que aparece en mayor porcentaje. Cuando el porcentaje de ambas adiciones es el mismo el comportamiento es prácticamente intermedio. Al ser diferente la evolución en el tiempo de la micro y la nano adición, las mezclas que contienen ambas van igualando su comportamiento, resultando a los 90 días un comportamiento intermedio al que se obtiene para el mismo porcentaje de cada adición. La mezcal con el 2,5 de nano y micro sílice presenta un comportamiento diferencial a edades largas. En esta caso su relación gel/ portlandita es superior a la que se obtiene utilizando un $5 \%$ de nano sílice. Este resultado es coherente con el comportamiento mecánico y durable de la mezcla. Este comportamiento podría indicar que en esta dosificación la relación entre la superficie de la adición y el agua libre ha resultado óptima.

\section{CONCLUSIONES}

La incorporación de nSi genera una pérdida de docilidad del material asociado a un aumento en la velocidad de fraguado. Fenómeno que dificulta su colocación “in situ”. La viscosidad y adaptación 
Revista ALCONPAT, Volumen 6, Número 1, Enero - Abril 2016, Páginas 1 - 14

al molde se dificulta notablemente cuando se incorpora a la masa nSi. Los hormigones con un contenido del $7.5 \%$ en $\mathrm{mSi}$ o con mezclas binarias [nmSi]-5/2.5; [nmSi]-2.5/5 se pueden considerar hormigones con capacidad autoniveladora. Sin embargo la adición de nSi como única adición, supone una pérdida de esta propiedad. La dificultad que puede tener el hormigón al paso con obstáculos aumenta a medida que es mayor la cantidad de nSi. Sin embargo en hormigones con $\mathrm{mSi}$ o con adiciones binarias se puede considerar que esta propiedad es independiente del contenido de dichas adiciones, aunque hay que señalar que son las mezclas con mSi las que presentan mejor comportamiento.

La incorporación de nSi genera un aumento significativo en la resistencia a compresión respecto a los hormigones con $\mathrm{mSi}$ y respecto al de referencia. La mezcla [nmSi]-2.5/2.5 es la que presenta valores más altos, esto puede deberse a una distribución granulométrica más continua considerando todos los componentes que contiene el hormigón, de manera que hay una menor formación de huecos y por tanto, se obtiene un hormigón más compacto. La incorporación de nSi, mSi o mezclas binarias de ambas adiciones producen, en los hormigones diseñados, un ligero aumento en el valor de tracción indirecta. Los hormigones con solo nSi presentan una disminución en su módulo de elasticidad, lo que conlleva mezclas con menor ductilidad.

Los hormigones con nSi presentan los mayores valores en las relaciones entre la pérdida de agua de gel y de la portlandita a las primeras edades, lo que pone de manifiesto que la formación de gel secundario o tobermorita es mayor cuando se utilizan nano adiciones. Así mismo, en el caso de los hormigones con $\mathrm{mSi}$, las relaciones presentan valores menores, resultado concordante con la menor resistencia que presentan. En mezclas binarias y con la misma cantidad total de adiciones y a largas edades, los valores de la relación se aproximan a los valores intermedios de las dos adiciones en la misma proporción El comportamiento singularmente bueno de la mezcla

[nmSi]-2.5/2.5 podría sugerir que existe una relación optima de superfice /agua libre que mejoraría el comportamiento de cualquiera de las dos adiciones estudiadas individualmente.

\section{AGRADECIMIENTOS}

Los autores agradecen el apoyo financiero del $\mathbf{M}^{\mathbf{o}}$ de Economía y Competitividad (España), Proyecto de investigación MAT2013-48009-C04-04-P.

\section{REFERENCIAS}

Bjornstrom, J.; Martinelli, A.; Matic, A.; Borjesson, L.; Panas, I.; (2004), "Efectos de la nanosílice coloidal en la aceleración de la formación beneficiosa de silicato de calcio hidratado en el cemento" (in English), Chem Phys Lett 392 (1-3), 242-248.

Borralleras, P. (2012), "Criterios de selección del aditivo superplastificante en HAC", $3^{\circ}$ Congreso Iberoamericano sobre hormigón autocompactante: Avances y oportunidades., 3-4 diciembre. Madrid. España.

Craeye, B.; Van Itterbeeck, P.; Desnerck, P.; Boel, V.; De Schutter, G.; (2014), "Módulo de elasticidad y tensión de tracción de un hormigón autocompactante: Encuesta de datos experimentales y códigos de diseño estructural" (in English), Cement and Concrete Composites 54, 53-61.

De la Peña R. Bernardo (2001), "Hormigón Autocompactante”, Revista BIT, pp. 7-12.

Dubey, R.; Kumar, P.; (2012), "Efecto de la dosis de superplastificante en la resistencia a compresión de un hormigón autocompactante" (in English), International Journal of civil, structural, environmental and infrastructure engineering research and development vol. 2, (3), pp 98-105 
Revista ALCONPAT, Volumen 6, Número 1, Enero - Abril 2016, Páginas 1 - 14

EHE-08. (2010), "Instrucción de Hormigón Estructural", Serie Normativa, Ministerio de Fomento. Secretaría General Técnica. 4ª Edición. Madrid. España.

Jalal, M.; Mansouri, E.; Sharifipour, M.; Pouladkhan, A. R.; (2012), "Propiedades mecanicas, reológicas, durables y microestructurales en un hormigón autocompactable de alta prestación conteniendo micro y nano partículas de $\mathrm{SiO}_{2}$ " (in English), Materials and Design 34, 389-400 Kawashima, S.; Hou, P.; Corr, D. J.; Shah, S. P.; (2013), "Modificación de materials base cement con nanopartículas" (in English), Cement and Concrete Composites. 36, 8-15.

León, N.; Massana, J.; Alonso, F.; Moragues, A.; Sánchez-Espinosa; E.; (2014), "Efecto de la nano-Si ${ }_{2} \mathrm{O}$ y nano- $\mathrm{Al}_{2} \mathrm{O}_{3}$ en morteros de cemento para uso en la agricultura y en la producción animal" (in English), Biosystems engineering 123, 1-11.

Mondal, P.; Shah, S. P.; Marks, L. D.; Gaitero, J. J.; (2010), "Estudio comparativo del efecto de la Microsilice y la Nanosilice en el hormigón" (in English), Transportation Research Record: Journal of the Transportation Research Board, $\mathrm{n}^{\mathbf{0}}$ 2141, Transportation Research Board of the National Academies, Washington, D.C., pp. 6-9.

Nazari, A.; Riahi, S.; (2010), "Comportamiento microestructural, térmico, físico y mecánico de un hormigón autocompactante conteniendo nanopartículas de $\mathrm{SiO}_{2}$ " (in English), Materials Science and Engineering A 527.7663-7672.

Okamura, H. Ozawa, K. (1996), "Hormigón autocompactante de altas prestaciones en Japón" (in English), ACI publicación especial SP159-02, pp. 31-44.

Okamura, H. (1997), "Hormigón autocompactante de altas prestaciones" (in English), Concrete International, 19 (7), pp. 50-54.

Okamura, H., Ouchi, M. (1999), "Desarrollo de un hormigón autocompactante, presente y futuro" (in English), Proceedings of the First International RILEM Symposium, pp. 3-14.

Okamura, H., Ozawa, K., Ouchi, M. (2000), "Hormigón autocompactante” (in English), Structural Concrete, 1, pp. 3-17.

Okamura, H., Maekawa, K., Mishima, T. (2005), "Diseño basado en las prestaciones para un hormigón estructural autocompactante de alta resistencia" (in English), ACI publicación especial SP228-02, pp. 13- 33.

Rong, Z.; Sun, W.; Xiao, H.; Jiang, G.; (2015), "Efectos de partículas de nano-SiO 2 en las propiedades mecánicas y microestructurales de composite cementoso de ultra-alta prestación" (in English), Cement and Concrete Composites 56, 25-31.

Said, A.M.; Zeidan, M.S.; Bassuoni, M.T.; Tian, Y.; (2012), "Propiedades del hormigón incorporando nano-silice" (in English), Construction and Building Materials 36. 838-844.

Sánchez, F. and Sobolev, K. (2010), "Nanotecnología en el hormigón - Una revisión” (in English), Construction and Building Materials. 24 2060-2071.

Yu, R.; Spiesz, P.; Brouwers, H.J.H; (2014), "Efecto de la nano-silice en la hidratación y desarrollo microestructural de un hormigón de ultra-altas prestaciones (HUAP) con una baja cantidad de conglomerante" (in English), Construction and Building Materials 65,140-150.

Zyganitidis; I.; Stefanidou, M.; Kalfagiannis; N.; Logothetidis S.; (2011), "Caracterización nanomecánica de pasta base-cemento enriquecidas con nanoparticulas de $\mathrm{SiO}_{2}$ ” (in English), Materials Science and Engineering B, 176, 1580-1584. 